\title{
Non-communicable disease management in vulnerable patients during Covid-19
}

\section{SAURAV BASU}

\section{Abstract}

It is now well established that non-communicable diseases $(N C D)$, like diabetes mellitus, hypertension,, respiratory and heart disease, particularly among the elderly, increase the susceptibility to Covid-19 disease. Mortality in 60\%-90\% of the Covid-19 cases is attributed to either one or more of these comorbidities. However, healthcare management for control of Covid-19 involves public health and policy decisions that may critically undermine the existing health needs of the most vulnerable NCD patients. Temporary closure of outpatient health facilities in some secondary and tertiary care hospitals have deprived millions of NCD patients of their regular medication and diagnostic health needs. The lack of robust primary healthcare facilities in most states, and the failure to maintain physical distancing norms due to inadequate infrastructure is also problematic. In the absence of effective public health interventions, socioeconomically vulnerable patients are likely to become non-adherent increasing manifold their risk of disease complications.

In this context, the feasibility of dispensing longer than usual drug refills for chronic NCD conditions at functional government health facilities, home delivery of essential drugs, running dedicated NCD clinics at PHCs, and utilisation of telemedicine opportunities for care and support to patients warrant aggressive exploration.

Keywords: Covid-19, NCDs, Medical ethics, epidemic, India

\section{Background}

The novel SARS-CoV2 since its origin in December 2019 in Wuhan, China continues to cause large scale morbidity and mortality globally, through a respiratory infection that is transmitted via droplet infection and causes pneumonia-like illness (1). The resultant Covid-19 pandemic-related worldwide burden as of April 19, 2020, stands at 23,74,141 positive cases and $1,63,272$ deaths (2).

It is now well established that non-communicable diseases (NCD) like diabetes mellitus, hypertension, respiratory (COPD / bronchial asthma), and heart disease, particularly among

Author: Saurav Basu (saurav.basu1983@gmail.com), Senior Resident, Department of Community Medicine, Maulana Azad Medical College, 2, Bahadur Shah Zafar Marg, New Delhi 110002 INDIA/

To cite: Basu S. Non-communicable disease management in vulnerable patients during Covid-19. Indian J Med Ethics. 2020 Apr-Jun; 5(2) NS:103-5, DOI:10.20529/JJME.2020.041.

Peer Reviewer: Mala Ramanathan

(๑) Indian Journal of Medical Ethics 2020 the elderly, increase the susceptibility to Covid-19 disease (3). According to global estimates, a high risk of mortality in Covid-19 cases is attributed to either one or more of these comorbidities $(4,5)$. Risk factors for NCDs like cigarette smoking and the harmful use of alcohol may also increase the susceptibility to Covid-19 disease.

However, there is growing recognition that the lack of guidelines for effective programmatic management of NCDs during this pandemic can hinder efforts in combating both these ongoing epidemics (6). Furthermore, in developing countries like India, which cumulatively account for $80 \%$ of the global NCD burden (7), the challenge of maintaining continuity of care in NCD patients at the time of the Covid-19 pandemic acquires enormous significance in the context of patient health outcomes and upholding public health ethics.

\section{Challenges in combined NCD and Covid-19 epidemic management in resource-constrained environments}

Ethical dilemmas in managing NCD conditions like diabetes mellitus in resource-constrained environments involve issues relating to lack of continuous medication supplies, diagnostics for follow-up, and associated clinical inertia or the failure to sufficiently intensify the medical therapy when the patient is not meeting his/her therapeutic targets.

Socioeconomically disadvantaged patients are most vulnerable for failing to meet their self-care, particularly medication adherence requirements (8). However, healthcare management for control of Covid-19 involves public health and policy decisions that may critically undermine the existing health needs of the most vulnerable NCD patients.

Efforts in containing the Covid-19 epidemic have required the strict implementation of lockdown and physical distancing requirements to prevent contagious patients coming in contact with the susceptible cohort. Similarly, in India, authorities have announced a temporary closure of outpatient facilities in government secondary and tertiary care facilities, while some of them have been converted into dedicated Covid-19 hospitals (9). However, millions of NCD patients across India are dependent on the public health system for medication refills that are needed for the management of their condition throughout their life. Government hospitals cater to a considerable proportion of such patients who may lack any other means of procuring their medication supplies (10). Patients with lower socioeconomic status (SES) are vulnerable to medication non-adherence and adverse health outcomes but the problem can be particularly exacerbated during the ongoing Covid-19 epidemic due to loss of jobs and wages coupled with disruption in their usual sources of 
drug access. Consequently, any additional out of pocket costs imposed on such patients can reach a catastrophic level. Nonadherent patients having NCDs have a manifold higher risk of complications resulting from uncontrolled disease. However, when patient non-adherence is unintentional, and due to lack of access or affordability of medication, it indicates a failure to uphold the principle of distributive justice through equitable distribution of resources.

Robust primary healthcare can be a valuable alternative resource for obtaining follow-up care and medications for patients with NCD belonging to low socioeconomic status. Nevertheless, a significant proportion of primary healthcare facilities in India have suboptimal infrastructure, which is not conducive to maintaining the physical distancing requirement of at least 1 metre between patients (11). These include the lack of multiple entry and exit points, limited seating and queuing facility, especially at a time of higher than usual patient load, and ineffective airborne infection control measures. These risks are likely to be accentuated in districts having hotspots, clusters, or during community transmission of Covid-19. NCD patients may, therefore, continue to be at persistent risk of Covid-19 while attending $\mathrm{PHCs} / \mathrm{CHCs}$ for meeting their health requirements. This undermines the principle of nonmaleficence or doing no harm to the patient by failure to prevent unnecessary exposure to the risk of disease.

The feasibility of these urgent corrective steps needs to be explored:

- Developing equitable solutions for population-based NCD management at the time of the pandemic is one option. Dispensing longer than usual drug refills for chronic NCD conditions at functional government health facilities must be considered. Public health facilities in India usually provide short duration medication drug refills ranging from 2 to 4 weeks to patients with NCDs (10). Dispensing prolonged duration refills with quarterly patient follow-up has well-established safety without any detrimental effect on patient health outcomes (12). However, arranging a significantly larger volume of drug inventory at short notice would also require a considerable mobilisation of scarce resources. Nevertheless, the benefits of decongesting outpatient health facilities, and achieving social distancing in immunocompromised and vulnerable patients who are highly susceptible to Covid-19 is ample justification for pursuing such a policy.

- The government can also utilise the vast network of its quality assured generic medicine distribution platform, the Jan Aushadhi Kendras for distribution of medications, while temporarily waiving costs if necessary for poor patients (13). Non-government organisations, health volunteers, and community health workers can also contribute towards the doorstep delivery of essential drugs for NCD management to the socioeconomically vulnerable patients, the elderly, and those lacking mobility and social support.

- Preventing patients seeking NCD care from coming in contact with patients reporting with flu like illnesses or suspected Covid-19 cases is essential for their protection. This would also ensure higher beneficence while reducing the risk of violating non-maleficence. Nevertheless, running dedicated NCD clinics can be problematic due to the risk involving overcrowding of patients. In this regard, assessing the feasibility of outpatient operations, which minimizes the risk of nosocomial novel coronavirus infection among patients with NCDs should be considered by the medical officer in charge of the individual health facility.

- Telemedicine can be leveraged for the provision of medical consultation to NCD patients in the absence of physical appointments for the alteration of their drug regimen, interpretation of blood investigations, and adherence support. These facilities can be explored across various audio-visual, telephonic, and internet-based mediums (14).

In conclusion, the failure to address and sufficiently resolve the barriers in attaining acceptable levels of care and management of patients having NCDs at the time of the Covid-19 pandemic represents a grave public health concern. The situation also highlights a unique intersection of public health ethics with the principles of biomedical ethics. Formulation of any relevant health policy must avoid neglect and tighten focus on this highly vulnerable population for the fulfilment of their essential health needs.

\section{Conflicts of interest and sources of funding: None}

\section{References}

1. Li Q, Med M, Guan X, Wu P, Wang X, Zhou L, et al. Early transmission dynamics in Wuhan, China, of novel coronavirus-infected pneumonia. NEngl J Med. 2020 Mar 26; 382(13):1199-207

2. Coronavirus Resource Centre. John Hopkins University \& Medicine. 2020 [Cited 2020 Apr 19]. Available from: https://coronavirus.jhu.edu/map. html

3. Fauci AS, Lane HC, Redfield RR. Covid-19 - Navigating the Uncharted. $N$ Engl J Med. 2020 Mar 26;382(13):1268-9.

4. Fang L, Karakiulakis G, Roth M. Are patients with hypertension and diabetes mellitus at increased risk for Covid-19 infection? Lancet Respir Med. 2020 Apr;8(4):e21.

5. Leung C.Clinical features of deaths in the novel coronavirus epidemic in China. Rev Med Virol 2020 Mar 16;e2103. doi: 10.1002/rmv.2103.

6. Thakur JS. Novel coronavirus pandemic may worsen existing global noncommunicable disease crisis. Int J Non-Commun Dis 2020;5(1):1-3.

7. Arokiasamy P. India's escalating burden of non-communicable diseases. Lancet Glob Health. 2018;6(12):e1262-3.

8. Basu S, Sharma N. Under-recognised ethical dilemmas of diabetes care in resource-poor settings. Indian J Med Ethics. 2018;3(4):324-6.

9. India Today. Delhi govt-run LNJP will function as dedicated Covid-19 hospital: Health Minister Harsh Vardhan. 2020 Apr 4 [cited 12.4.2020] Available from: https://www.indiatoday.in/india/story/coronavirusindia-Injp-hospital-function-dedicated-Covid-19-health-ministerharsh-vardhan-1663376-2020-04-04

10. Basu S, Garg S, Sharma N, Singh MM, Garg S, Asaria M. The determinants of out-of-pocket health-care expenses for diabetes mellitus patients in India: An examination of a tertiary care government hospital in Delhi. Perspect Clin Res [Epub ahead of print] [cited 2020 Apr 12]. Available from: http://www.picronline.org/preprintarticle.asp?id=257232

11. Sriram S. Availability of infrastructure and manpower for primary health centers in a district in Andhra Pradesh, India. J Family Med Prim Care 2018;7(6):1256-62.

12. Bahrani K, Singh MB, Bhatia R,Prasad K,Vibha D, Shukla G, et al.Telephonic review for outpatients with epilepsy; a prospective randomized, parallel group study. Seizure. 2017 Dec; 53:55-61.

13. Janaushadhi.gov. India: Introduction to Jan Aushadhi. [cited 12.4.2020] 
Available from: http://www.janaushadhi.gov.in/about_jan_aushadhi. html.

14. Chauhan V, Galwankar S, Arquilla B, Garg M, Somma SD, El-Menyar A,
Krishnan V, Gerber J, Holland R, Stawicki SP. Novel coronavirus (Covid-19): Leveraging telemedicine to optimize care while minimizing exposures and viral transmission. J Emerg Trauma Shock 2020;13:20-4.

\section{Alcohol withdrawal management during the Covid-19 lockdown in Kerala}

\section{RAVI PRASAD VARMA}

\begin{abstract}
The lockdown declared to prevent the spread of Covid-19 in India created unforeseen problems, including severe alcohol withdrawal symptoms and the need to manage them. The state of Kerala in India saw suicide deaths by six affected individuals, prompting the state government to instruct government doctors to prescribe alcohol to addicts. The local medical association approached the courts against this. These events raise interesting ethical issues discussed here.
\end{abstract}

Keywords: alcohol withdrawal, prescribing alcohol, Covid-19, ethics of alcohol prescription.

Alcohol is often considered a social evil, a poison which is bad for your health. So what prompted the government of Kerala to instruct doctors to prescribe alcohol to those suffering from its withdrawal? It began with the total lockdown of almost all non-essential services including liquor stores, against the threatening surge of Covid-19. The declaration was sudden and left everyone in the lurch, tipplers included. Kerala started witnessing a small wave of suicides attributed to alcohol withdrawal, with six such deaths as against two attributed to Covid-19, as on April 1, 2020 (1). In response, the concerned State authorities issued an order directing those with alcohol withdrawal symptoms to approach doctors at government hospitals for "passes" which would help them procure alcohol (2). The order immediately met with resistance from doctors. Leading professional medical organisations promptly obtained a stay from the Kerala High Court against the order (3). The predicament of those with alcohol withdrawal symptoms, the state's attempt to offer relief, and the doctors' unobliging rejoinder raise interesting ethical questions.

Persons who drink alcohol in considerable amounts over a long period of time are likely to become "dependent" on alcohol. This means that they develop some physical or mental reactions if they stop drinking. Some may experience only minor symptoms like shaking hands, headache, nausea and vomiting. In others these symptoms can be severe, resulting

Author: Ravi Prasad Varma (rpvarma@sctimst.ac.in), Associate Professor, Achutha Menon Centre for Health Science Studies, Sree Chitra Tirunal Institute for Medical Sciences and Technology, Trivandrum.

To cite: Varma RP. Alcohol withdrawal management during the COVID-19 lockdown in Kerala. Indian J Med Ethics. 2020 Apr-Jun; 5(2) NS: 105-6. DOI:10.20529/JJME.2020.042.

() Indian Journal of Medical Ethics 2020 in seizures or delirium tremens with high fever, high blood pressure, confusion, and hallucinations, and may even be fatal. The reported suicides of young persons testify to the gravity of these people's suffering under the current lockdown.

What are the rights of such habitual users of this statesanctioned (albeit controlled) substance under a lockdown? The government's response to the issue clearly indicates the State's acceptance of its responsibility for the problem and this aspect is commendable. The relationship of a state to alcohol is determined by historical and contemporary values around alcohol use in society. Alcohol is often a masculine motif and societies with strong patriarchal mores may add impetus to the state's position on alcohol. Another important aspect is the economics of the production, distribution and consumption of the substance. Stringent control measures may lead to illicit liquor production and consumption with disastrous health consequences. Thus, many governments support controlled availability of alcohol, aiming for a judicious balance between use and abuse.

Was it right on the part of the medical professionals, especially those employed by the state, to refuse to "prescribe" passes for persons with symptoms of alcohol withdrawal? Alcohol can cause severe medical illness - most medical students encounter the big belly of alcoholic liver disease early on in their careers. Medical lexicons are populated with many alcohol related diseases - alcoholic polyneuropathy, alcoholic myopathy, alcoholic cardiomyopathy, foetal alcohol syndrome and several more. It is easy to understand the doctors' opposition to this state order given the overall ethos of the profession in this regard. Additionally, the order has been interpreted as impinging on the right of medical practitioner to determine the contents of a prescription, given that there are effective treatments for symptoms of alcohol withdrawal. Doctors are also working in a situation where optional medical consultations including vaccinations are heavily restricted; and a call for alcohol prescriptions at such a time does raise a morally loaded question for many doctors.

Clearly, there is no simple answer to this dilemma. Should we let those in withdrawal pay the price in the interest of the greater good - prevention of rapid surge or better mitigation of the Covid-19 outbreak? They are after all part of the larger societal phenomenon. Will it be possible for the state or the health system to make the necessary treatments available where it is needed at a time like this? Such situations may test the harmony between various stakeholders at the forefront 